\title{
CHRONIC COR PULMONALE
}

\author{
M. K. Towers, M.A., M.B., M.R.C.P. \\ Cardiologist, Harefield and Clare Hall Hospitals \\ Research Assistant, Institute of Diseases of the Chest
}

\section{Definition}

Chronic Cor Pulmonale is defined as right ventricular hypertrophy due to impairment of the ventilatory and respiratory function of the lungs. This is a narrower definition than that of the World Health Organisation (1961) and excludes primary diseases of the lung vasculature which have a different physiological mechanism and clinical course.

\section{Diseases causing Chronic Cor Pulmonale}

The diseases causing Chronic Cor Pulmonale listed by the World Health Organisation (1961) are :-

I. Diseases primarily affecting air passages of the lung and the alveali.

1. Chronic bronchitis with generalized airways obstruction with or without emphysema.

2. Bronchial asthma.

3. Emphysema without bronchitis or asthma.

4. Pulmonary fibrosis, with or without emphysema, due to :-

(a) Tuberculosis

(b) Pneumoconiosis

(c) Bronchiectasis

(d) Other pulmonary infections

(e) Radiation

(f) Muco-viscidosis

5. Pulmonary granulomata and infiltrations
(a) Sarcoidosis
(b) Chronic diffuse interstitial fibrosis
(c) Berylliosis
(d) Eosinophilic granuloma or histio- cytosis
(e) Malignant infiltration
(f) Scleroderma
(g) Disseminated lupus erythematosus
(h) Dermatomyositis
(i) Alveolar microlithiasis

6. Pulmonary resection.

7. Congenital cystic disease of the lungs.

8. High altitude hypoxia.

II. Diseases primarily affecting the movements of the thoracic cage

1. Kyphoscoliosis and other thoracic deformities

2. Thoracoplasty

3. Pleural fibrosis

4. Chronic neuromuscular weakness-e.g., poliomyelitis

5. Obesity with alveolar hypoventilation

6. Idiopathic alveolar hypoventilation

\section{Pathogenesis}

In the following discussion and in the account of the clinical and other findings attention will be concentrated on bronchitis and emphysema which $\vec{N}$ are the most important causes of pulmonary heart disease.

Cor pulmonale is always associated with disturbances in the blood gases and a number of $\%$ mechanisms are involved. Chronic bronchitis and 5 emphysema are characterised by airways obstruction. In bronchitis it is due, partly at least, to excessive secretion of mucus and in emphysema 0 to the expiratory collapse of poorly supported small airways. Obstructed airways limit the rate at $\overrightarrow{\vec{\theta}}$ which air can move in and out of those parts $\mathscr{\&}$ the lungs which they serve; many alveoli are. underventilated or not ventilated at all. There grossly uneven matching of ventilation to blood flow in the lung; parts of the lung are well perfused but poorly ventilated, whilst others are well ventilated but poorly perfused. The well $\frac{\mathrm{D}}{\mathrm{D}}$ ventilated areas cannot compensate entirely for 2 the poorly ventilated; alveolar hypoventilation $\overrightarrow{\bar{O}}$ invariably results in hypoxia, hypercapnia and $\exists$ acidosis, and the overventilated alveoli cannot $\supset$ supply a sufficient excess of oxygen to counterbalance the hypoxia.

In pulmonary fibrosis and in the pulmonary granulomata, ventilation-perfusion inequalities are $\dot{\sigma}$ the main cause of blood gas changes. Kypho- 3 scoliosis has been studied by Fishman (1965). $\delta$ Gross distortion of the chest increases the energy cost of breathing, and rapid shallow breathing 0 (the most economical pattern) is adopted. This $]$ results in good ventilation of the dead space but $\frac{D}{O}$ poor ventilation of the alveoli and leads to blood gas changes. In the Pickwickian syndrome (obesity N with alveolar hypoventilation) diminished respira-o tory drive is the most important abnormality.

Thus, a great variety of ventilatory and respira- N tory faults can result in blood gas disturbances. O In a patient with moderately severe bronchitis ${ }_{0}$

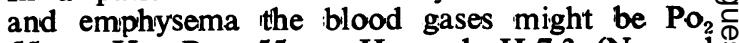
$55 \mathrm{~mm} . \mathrm{Hg}$., $\mathrm{PcO}_{2} 55 \mathrm{~mm} . \mathrm{Hg}$. and $\mathrm{pH}$ 7.3. (Normal $\stackrel{\oplus}{+}$ values $\left.\mathrm{Po}_{2} 100, \mathrm{PcO}_{2} 40, \mathrm{pH} 7.4\right)$. It is difficult to sort out the separate effects of anoxia, ${ }_{0}$ hypercapnia and acidosis on individual organs. 
The sum of these effects and their interaction on various organs and systems are the manifestations of cor pulmonale. The lung vessels, the kidney, the haemopoietic system, the brain, the peripheral circulation, the liver and the heart are principally involved.

There has been doubt about the relative importance of organic and functional changes in the lung vasculature. Widespread loss of the pulmonary vascular bed is an important cause of pulmonary hypertension in some cases of emphysema. Nevertheless, Fletcher, Hugh-Jones, MoNichol and Pride (1963), have shown that cor pulmonale occurs more often with chronic bronchitis than with emphysema. The partial reversibility of cor pulmonale also suggests functional rather than organic change in the lung vessels and contrasts with inexorable deterioration in obliterative pulmonary hypertension. Vasoconstriction brought about by the interaction of low alveolar oxygen tension and high blood hydrogen ion concentration upon muscular pulmonary arteries of about $1 \mathrm{~mm}$. in diameter (Harvey, 1965) is the most important cause of pulmonary hypertension. Of course, as in systemic hypertension, continual vasoconstriction can give rise to secondary organic changes in the vessels.

Blood gas disturbances probably cause both renal vasoconstriction and alterations in tubular function. The renal blood flow was low in all the patients studied by Aber, Bailey and Bishop, (1963), even when the cardiac output was normal. This is probably due to renal vasoconstriction. In oedematous patients the low glomerular filtration rate alone seemed insufficient to account for the excessive sodium and water retention, so that tubular function is probably altered.

Shaw and Simpson (1961) have shown that in emphysema the marrow is stimulated probably by tissue anoxia to produce more red blood cells. This may lead to polyoythaemia with an increased haematocrit, but more often there is a concomitant increase in plasma volume so that the haematocrit is normal even though the red-cell mass is increased.

Elevation of $\mathrm{Pco}_{2}$ dilates the cerebral vessels, raises the pressure of the cerebrospinal fluid causing headache, drowsiness and in extreme cases papilloedema (Hamilton and Gross, 1963). A sharp rise in $\mathrm{Pco}_{2}$ seems to be more important than the absolute level, but Campbell (1965) has pointed out that the more severe symptoms and really high $\mathrm{Pco}_{2}$ levels are not found unless oxygen has ibeen given. Hypoxia and not hypercapnia is the stimulus for the respiratory drive in these patients. If hypoxia is relieved by giving high concentrations of oxygen, the $\mathrm{PcO}_{2}$ builds up rapidly and carbon dioxide narcosis supervenes.

Peripheral vasodilation causing the full.bounding pulse and warm skin characteristic of pulmonary heart disease is attributed to rises in $\mathrm{PCO}_{2}$ (Hamilton and Gross, 1963). Thomas, Rees and Saunders $(1965)$ have showh that liver function is disturbed in pulmonary heart disease before the onset of "congestive failure". Hypoxia may be the cause of the liver dysfunction.

The cardiac output in pulmonary heart disease has been extensively studied. (Wade and Bishop, (1962), reviewing the literature, concluded that the cardiac output is normal and falls only in the late stages of the disease. Alber and others (1963) have shown that the cardiac output may bo normal oven in the oedematous patient. By contrast the cardiac output is low in most other kinds of "heart failure". This paradox highlights our present ignorance of the fundamental physiology of "heart failure". The term "congestive failure" is here used to describe a clinical syndrome without implying a cause.

A number of adverse factors affect the heart in cor pulmonale. Right ventricular work is increased because the cardiac output is maintained and there is pulmonary hypertension, even though this is moderate (systolic 40 to $100 \mathrm{~mm} . \mathrm{Hg}$.) rather than severe. If functional tricuspid incompetence supervenes, the right ventricular work is further increased. Raised blood viscosity in polycy themia also inoreases cardiac work. The effects of anoxia on the myocardium are difficult to assess, but must be unfavourable and may account for left ventricular hypertrophy found in some patients with pulmonary heart disease in whom there is no evidence of coronary artery disease or hypertension (Fluck, Chandrasekar and Gardner, 1966).

\section{Clinical Findings}

Typically, the patient is a middle aged man with a long history of ibronchitis and recurren't chest infections. An infection or a period of smog or cold weather causing an increase in cough, sputum and breathlessness and perhaps the appearance of ankle oedema, bring the patient to the doctor, Cough syncope, headache, drowsiness and palpitation are less common symptoms.

The patient is cyanosed and the warm skin indicates central and not peripheral cyanosis. There may or may not be clubbing of the fingers. The chest is held in the inspiratory position and is hyper-resonant. Usually there are expiratory wheezes and coarse crepitations. The jugular venous pressure is normal or raised if there is "congestive failure". The ' $a$ ' and ' $v$ ' waves are equal. The arterial pulse is of full volume and rapid in acute exacerbations of cor pulmonale. Sometimes an overactive right ventricle can bo felt and $\mathrm{P}_{2}$ ('the sound of pulmonary valve closure) may be palpable. More of ten the cardiac impulses are impalpable and the heart sounds distant because the lungs partly cover the heart. On auscultation $P_{2}$ may be increased but often it cannot be heard and a right atrial gallop, best heard at the lower end of the sternum, is the only sign. The gallop is characteristic because it is much louder on inspiration, not only because the atrial sound becomes louder but also because 


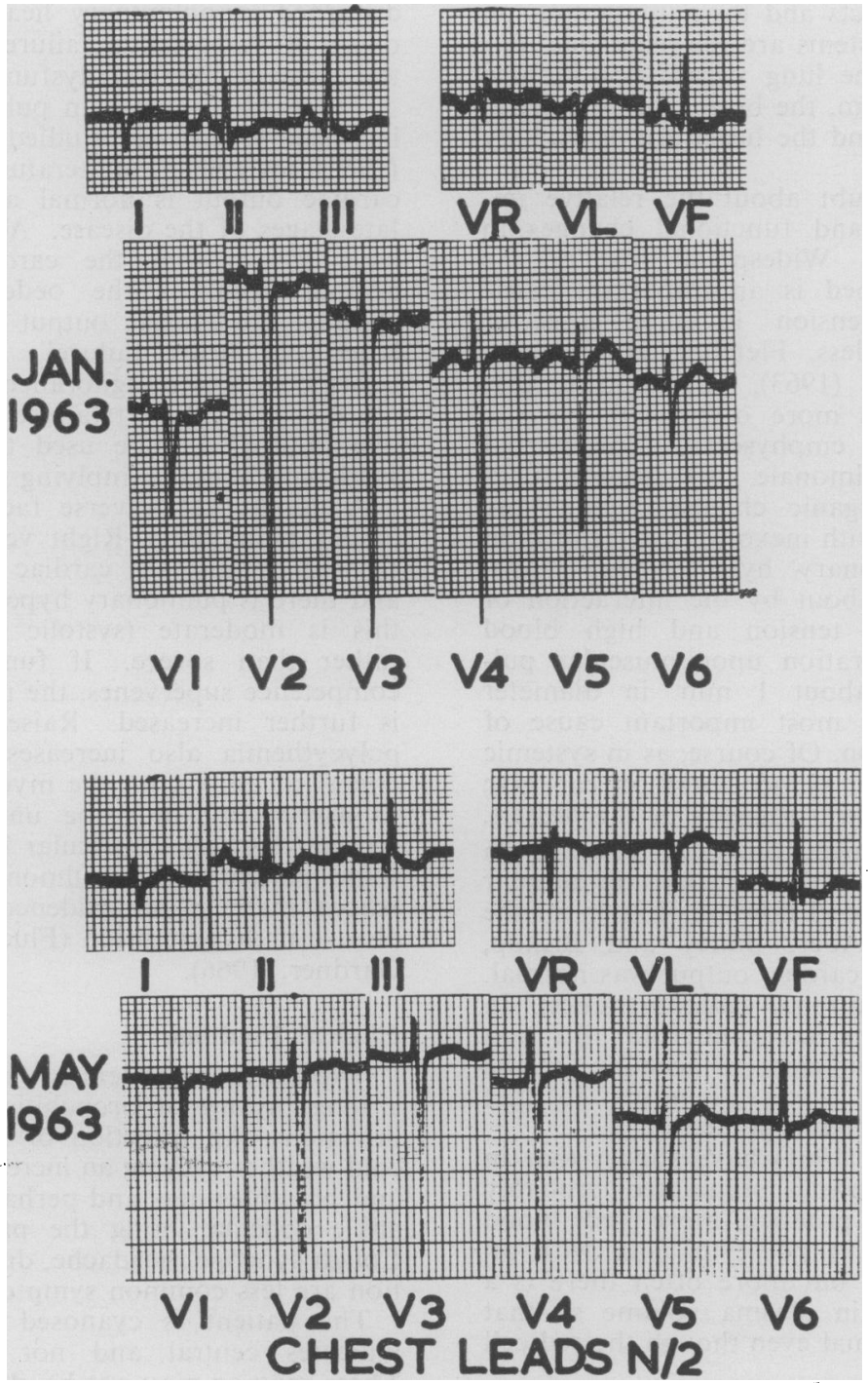

FIG. 1-Electrocardiograms during and after an acute chest infection in a patient with Cor pulmonale.

In the upper tracing, sinus tachycardia, $\mathbf{P}$ pulmonale, right axis deviation $(+110), r S$ pattern across the chest and $T$ wave inversion over the right chest. After treatment the rate is slower, the right axis deviation less (+95), the $\mathrm{T}$ wave is upright over V 2 and 3, and the rS pattern does not extend across the chest.

it tends to move away from the first sound and is more easily heard. In more severe cases with gross oedema the atrial gallop is replaced by a tricuspid pansystolic murmur and third sound. After treatment the atrial gallop becomes softer or disappears and the peripheral circulation returns to normal.

\section{Electrocardiogram}

The electrocardiographic signs of cor pulmonale are a $\mathbf{P}$ pulmonale, right axis deviation of the $\varphi$ mean QRS frontal vector (or occasionally an $S_{1}$, Ф $S_{2}, S_{2}$ pattern) and

either an $\mathrm{rS}$ pattern in the chest leads (the 0 commonest pattern). In a less fully ${ }_{0}$ developed form there is an Rs pattern 

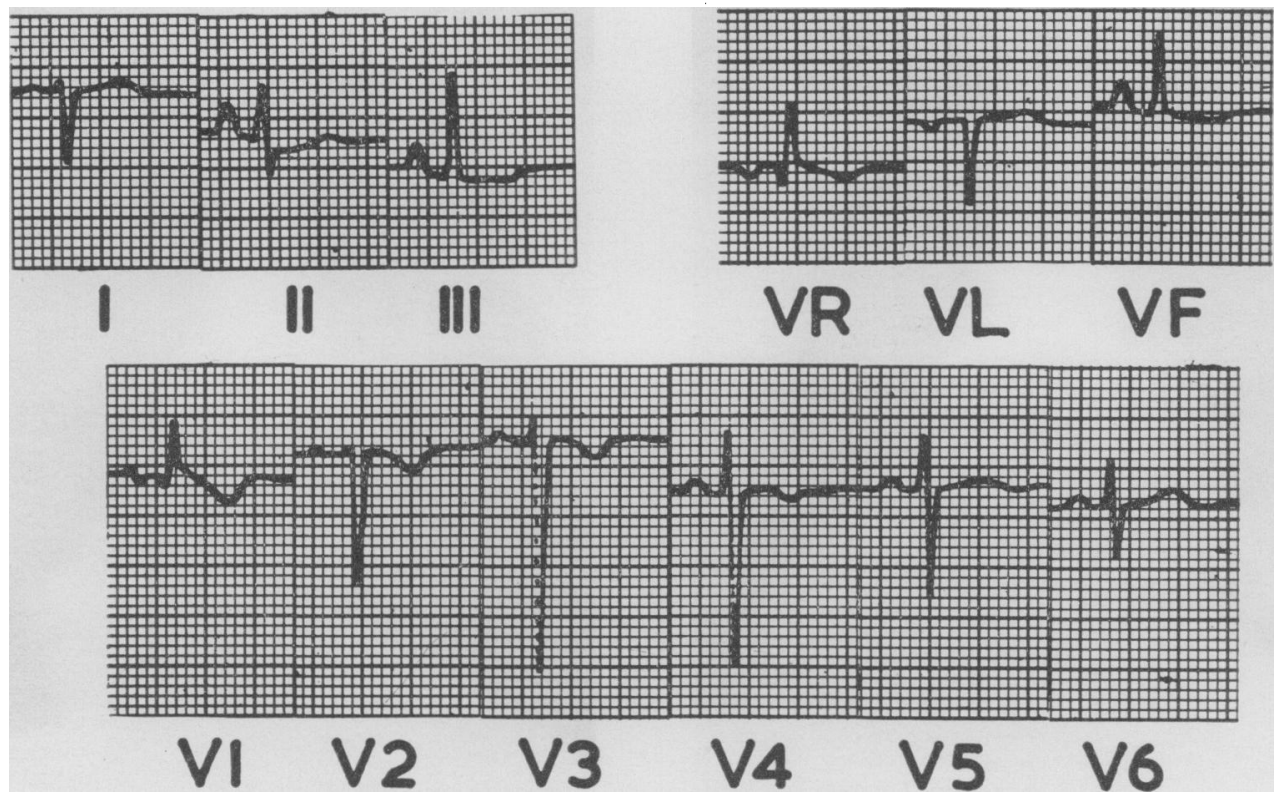

Fig. 2-Classical right ventricular hypertophy pattern in Cor pulmonale. $\mathbf{P}$ pulmonale, right axis deviation $(+110)$, dominant $R$ in $V 1$, inverted T's over the right chest and conspicuous $S$ waves in V6.

over the left chest. The $T$ wave is usually upright in the right chest leads but may be inverted during exacerbations.

or a classical right ventricular hypertrophy pattern with a dominant $R$ or $R^{1}$ in $V 1$ and inverted $T$ waves in the right chest leads.

or

right ibundle branch iblock.

Right axis deviation is due to right ventricular hypertrophy consequent on pulmonary hypertension and the $\mathbf{P}$ pulmonale indicates right atrial hypentrophy due to increased filling resistance of the hypertrophied right ventricle. The rS pattern in the chest leads is thought to be the result of changes in the position of the heart relative to the chest electrodes. The heart is rotated about its long axis by hypertrophy of the right ventricle and overinflation of the lungs may lower the diaphragm and heart in relation to the chest electrodes.

The most satisfactory evidence of right ventricular hypertrophy is an increase in the weight of the right ventricle when it has been dissected from the heart. There is good correlation between right ventricular weight and right axis deviation and $P$ pulmonale in the electrocardiogram recorded before death (Millard, 1966), but the cause of the $\mathrm{rS}$ pattern remains obscure.

The diagnosis of cor pulmonale can be made with confidence if the clinical circumstances are appropriate and one of the classical electrocardio- graphic patterns is found. A combination of right axis deviation and a $\mathbf{P}$ pulmonale alone is not conclusive evidence of right ventricular hypertrophy. This pattern can occur in very severe asthmatic attacks and resolve completely in a day or two, so that it can indicate right ventricular stress rather than hypertrophy. Many patients go through the clinioal course of their disease with an $\mathrm{rS}$ pattern in the chest leads. In others, over a long period, there is a transition to a classical right ventricular hypertrophy pattern which may indicate more severe pulmonary hypertension.

Co-existing ischaemic heart disease may make the electrocardiographic diagnosis of cor pulmonale difficult, especially by altering the mean QRS frontal vector. Using conventional electrocardiographic criteria Rees, Thomas and Rossiter (1964), showed that pulmonary heart disease could be diagnosed accurately in $72 \%$ of patients, coronary heart disease in $84 \%$ and both in $55 \%$. Grant (1957) suggests that overinflation of the lungs may cause electrical forces to run in a more vertical direction through the better-conducting mediastinum and thus give rise to left axis deviation. This idea is unproven and, of course, many of these patients are in the coronary age group.

A patient with cor pulmonale may be seen for the first time in a state of vasomotor collapse and pulmonary embolism has to be considered. A P pulmonale is more often seen in cor pulmonale; the $P$ wave axis tends to be $+70^{\circ}$ or more, an RS pattern is more often seen in the left sided 


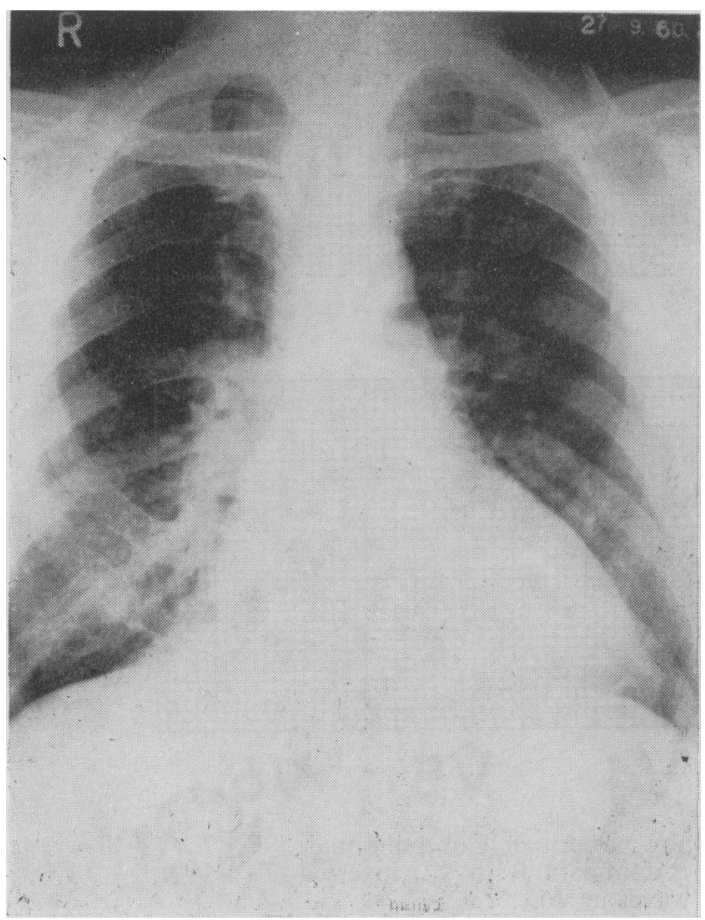

FIG. 3-Cor pulmonale with chest infection. Large heart, prominence of pulmonary artery and of right main branch. Infective shadow at right base. Right dome of diaphragm at level of sixth rib.

chest leads and the $T$ wave tends to be upright in the right chest leads (Caird and Stanfield, 1962). In the presence of cor pulmonale the electrocardiographic diagnosis of pulmonary embolism is almost impossible.

Disturbances of rhythm are not uncommon in cor pulmonale. Atrial ectopic beats are often seen and sometimes prolongation of the P-R interval. Atrial fibrillation is rare but paroxysmal atrial tachycardia with block occurs (Corazza and Pastor, 1958) usually in patients who are very ill. The possibility of digitalis-induced arrhythmia should be kept in mind.

\section{Chest X-ray}

Pulmonary heart disease causes enlargement of the right heart and pulmonary artery. In emphysema the heart shadow may be abnormally small and early enlargement is only discernible if a previous film is available for comparison. In the bronchitic patient with cor pulmonale an acute chest infection may cause considerable enlargement of the heart. When the infection is controlled the heart shadow may return almost to its original size.

The radiology of emphysema has been reviewed by Simon (1964). The signs are (i) Excess of air in the lungs shown by a low flat diaphragm (the central portion of the right dome being on

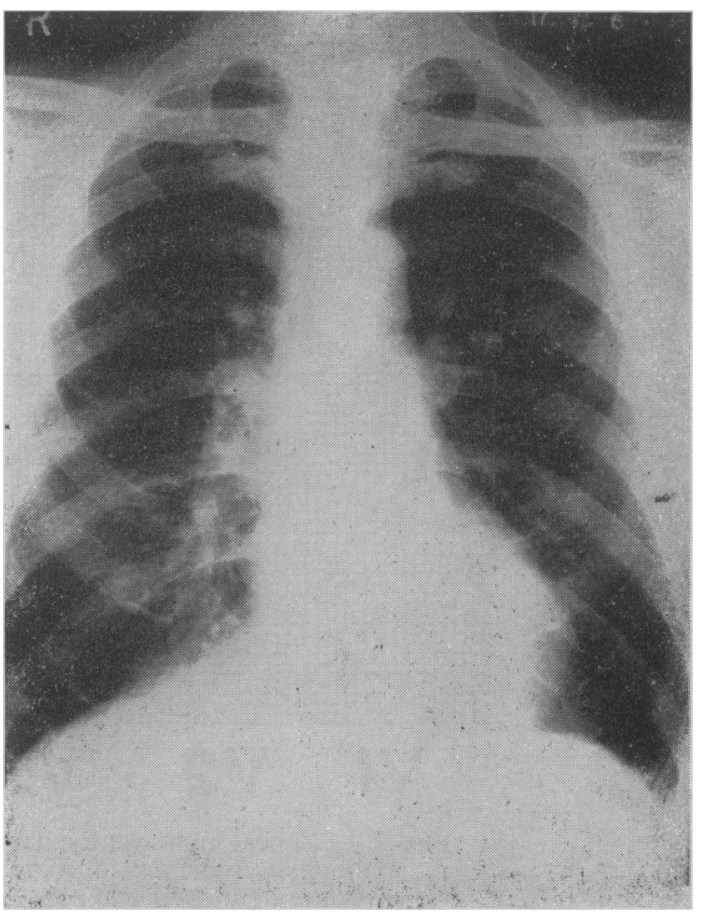

FIG. 4-Same case after treatment. Heart smallero Right dome of diaphragm below level of sevent rib. Hypertranslucent area at left base.

inspiration at or below the seventh rib anteriorly), and a large retrosternal translucent area seen in the lateral film. (2) Cardiovascular changes. The heart is narrow and vertical. Slight prominence of the main pulmonary artery is due sometimes to partial rotation of the heart, and sometimes to dilatation of the artery from pulmonary hypertension or surges in pressure on effort. There is a combination of large hilar vessels and small mid-lung vessels. (3) The presence of bullae or bullous areas. A patient with emphysema severe enough to cause pulmonary heart disease will show two or all three of the X-ray features. When a patient with emphysema develops oedema the heart enlarges and the diaphragm rises so that the radiological diagnosis of emphysema may not be obvious but it can readily be made from previous films if they are available.

There are no specific X-ray changes in bronchitis. Bronchograms if available may show enlarged ducts of mucus glands, peripheral nonfilling and dilatation of the terminal bronchioles. Other diseases causing pulmonary heart disease may present more specific appearances.

\section{Treatment}

Cor pulmonale is a late stage in the natural history of chest dísease; usually bronchitis and emphysema. Though dramatic improvements may 
be achieved in exacerbations, the disease is progressive and incurable. Effort should therefore be concentrated on arresting bronchitis at an earlier stage. The excessive secretion of mucus characteristic of bronchitis is maintained largely at least, by bronohial irritation. Smog, smoke, industrial and traffic fumes are important irritants and should be avoided, but for the majority of patients cigarette smoking is much the most potent and constant bronchial irritant. The patient should understand clearly the danger of smoking. He should be encouraged to give it up and where possible attendance at an anti-smoking clinic may help (Ball, Kirby and Bogen, 1965). Chest infections should be treated promptly and $H$. influenzae, the pneumococcus and oocasionally the staphylococcus are usually involved (May 1965). Ampicillin and tetracycline are the most useful drugs, and should be continued indefinitely if the sputum becomes purulent when treatment is stopped. Physiotherapy may be helpful especially when it can be arranged for groups of bronchitic patients.

The patient presenting with a chest infection, cor pulmonale and oedema, requires treatment for respiratory failure and for congestive failure. The detailed treatment of respiratory failure is outside the scope of this anticle. The most important measures are the administration of oxygen by Venturi mask (limiting the concentration to $25-29 \%$ of oxygen to avoid carbon dioxide narcosis), appropriate antibiotics, respiratory stimulants such as nikethamide intravenously, bronchodilators, steroids, physiotherapy and if necessary tracheostomy and assisted respiration. Digitalis and diuretics are given as for congestive failure. Digitalis is of marginal value but is probably worthwhile giving for its inotropic effect. Great care should be taken to avoid digitalis toxicity to which the elderly patients are especially liable (Schott 1964). If major disturbances of atrial rhythm ocour in the undigitalised patient, digitalis should be given. Strenuous attempts to restore normal rhythm with drugs or D.C. shock are often unsuccessful. When they are successful the improvement in the patient is apt to be disappointing. If digitalis is suspected as the cause of the arrhythmia propranolol is the drug of choice.

\section{Complications and Prognosis}

Two important complications of therapy, carbon dioxide narcosis and digitalis intoxication, have been discussed. A third, cerebral oedema, though directly due to cerebral hypoxia may be brought about by too rapid a lowering of the Pcos (Hamilton and Gross, 1963). Cerebral vasodilation produced by hypercapnia protects the brain, partly at least, from hypoxia. The rapid abolition of this vasodilatation leaves the brain especially vulnerable to hypoxia and irreversible coma or hemiplegia may follow the cessation of oxygen treatment. A further unusual complication of pulmonary heart disease described by Hamilton and Gross (1963) is pulmonary artery thrombosis in situ, probably the result of pulmonary vascular disease from longstanding pulmonary hypertension. It should be suspected if a patient with pulmonary heart disease develops intractable congestive failure in spite of improving respiratory function.

In the patient with "pure" bronchitis congestive failure is usually precipitated by a respiratory infection. The patient responds well to treatment and may be able to return to work. Several episodes of congestive failure may occur over a period of years, the patient being slightly worse after each one. In contrast, the patient with "pure emphysema" often develops congestive failure after a long period of slow deterioration and responds poorly to treatment (Fletcher and others, 1963). Presumably this is because of the larger irreversible element of pulmonary vascular damage in the emphysematous patient.

\section{REFERENCES}

Aber, G. M., BaIley, T. J., and Bishop, J. M. (1963): Inter-relationships between Renal and Cardiac Function and Respiratory Gas Exchange in Obstructive Airways Disease, Clin. Sci., 25, 159.

BALl, K. P., KIRBY, B. J., and BOGEN, C. (1965): First Year's Experience in an Anti-smoking Clinic, Brit. med. $J$., i, 1651.

CaIRD, F. I., and STANFIEld, C. A. (1962): The Electrocardiogram in Asphyxial and in Acute Embolic Cor Pulmonale, Brit. Heart J., 24, 313.

CAMPBell, E. J. M. (1965): Respiratory Failure, Brit. med. J., i, 1451 .

CoRrazza, L. J., and Pastor, B. H. (1958): Cardiac Arrhythmia in Chronic Cor Pulmonale, New Engl. J. Med., 259, 862.

FishmaN, A. P. (1965): Pulmonary Aspects of Scoliosis. Proceedings of a Symposium on Scoliosis (Ed. P. A. Zorab), p. 79. London: National Fund for Research into Poliomyelitis and other Crippling Diseases.

Fletcher, C. M., HuGH-JONES, P., MCNichol, M. W., and PRIDE, N. B. (1963): The Diagnosis of Pulmonary Emphysema in the Presence of Chronic Bronchitis, Quart. J. Med., 32, 33.

FlUCK, D. C., ChandRasekar, R. G., and Gardner, F. V. (1966): Left Ventricular Hypertrophy in Chronic Bronchitis, Brit. Heart J., 28, 92.

GraNT, R. P. (1957): CClinical Electrocardiography: The Spatial Vector Approach, p. 134. New York: McGraw-Hill Book Co.

Gross, N. J., and Hammton, J. D. (1963): Correlation between the Physical Signs of Hypercapnia and the Mixed Venous Pco2, Brit. med. J., ii, 1096.

HAMILTON, J. D., and Gross, N. J. (1963): Unusual Neurological and Cardiovascular Complications of Respiratory Failure, Brit. med. J., ii, 1092.

HARVEY, R. M. (1965): The Influence of Hydrogen Ion in the Control of Pulmonary Artery Pressure in Patients with Obstructive Disease of the Lungs. Progress in Research in Emphysema and Chronic Bronchitis (Ed. R. S. Mitchell), p. 108. Basel (Switzerland): S. Karger.

MAY, J. R. (1965): The Bacteriology and Chemotherapy of Chronic Bronchitis, Brit. J. dis. Chest, 59, 57. 
Millard, F. J. C. (1966): Brit. Heart J., to be published.

ReEs, H. A., Thomas, A. J., and Rossiter, C. (1964): The Recognition of Coronary Heart Disease in the Presence of Pulmonary Disease, Brit. Heart J., 26, 233.

Schotт, A. (1964): Observations on Digitalis Toxicity-a Plea, Postgrad. med. J., 40, 628.

Shaw, D. B., and Simpson, T. (1964): Polycythemia in Emphysema, Quart. J. Med., 30, 135.

SimON, G. (1964): Radiology and Emphysema, Clin.
Radiol., 15, 293.

Thomas, A. J., ReEs, H. A., and Saunders, R. A. (1965): Liver Function in Pulmonary Heart Disease, Brit. $\stackrel{\mathbb{1}}{\circ}$ Heart J., 27, 791.

WADE, O. L., and Bishop, J. M. (1962): Cardiac Output and Regional Blood Flow. Oxford: Blackwell Scientific Publications.

World Health Organisation Technical Report Series No. 213. (1961): Chronic Cor Pulmonale. 흘 Geneva: W.H.O.

\title{
TRIGGER THUMB IN INFANCY
}

\author{
A. P. Patel, F.R.C.S.E. \\ Department of Orthopaedics, The West Hill Hospital, Dartford, Kent
}

STENOSING tenovaginitis of the flexor tendons of the hand causing trigger or snapping finger in adults is a well recognised condition, but in young children is often misdiagnosed.

Tubby (1912) considered it to be congenital, Whitman (1927) described it as "not infrequently seen in infancy," and it has been described by Jahss (1936), Zadek (1942), Rose (1946), Spreecher (1949), Fahey and Bollinger (1954).

\section{Clinical features}

Ten young children with trigger thumbs were seen at The West Hill Hospital, Dartford, ranging in age from six months to four years, and of these, two were bilateral and eight were unilateral. A history of trauma was often given by the parents but in this series it was thought that the injury was incidental and had merely drawn attention to an already existing deformity. In one child with both thumbs affected, the deformity was noticed by the mother from birth. In the rest, it was present from six to twelve weeks prior to the parents seeking medical advice.

All the children presented with a flexion deformity of 15 to 50 degrees on the interphalangeal joint of the thumb, and attempts to correct it by force only caused pain. The other constant finding was the presence of a distinct palpable nodule over the volar surface of the neck of the first metacarpal. In none of the children could snapping or triggering be obtained, (Fukase, Fukada and Yamaguchi, 1961).

\section{Pathology}

Normally, the tendon sheath of the digit at the level of the metacarpophalangeal joint is slightly

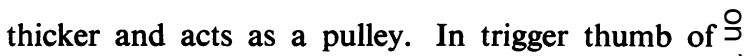
adults, this pulley is constricted and thickened and produces enlargement of the tendon on each side. The pathological lesion seems to be different in children, and in five children operated on in the last twelve months, no such undue thickening of tise $\vec{\ominus}$ pulley was found. Instead, a large bulbous nodure in the tendon was met with at this level. Distal this nodule, the pulley appeared to be constricted but this was rather relative to the size of the tendon.

Biopsy of the tendon nodule showed normal tendon tissue infiltrated with lymphocytes and mono- $\stackrel{\mathbb{D}}{\mathbb{2}}$ cytes (Spreecher, 1949), and evidence of colliquative degeneration (Fahey and Bollinger, 1954). This is consistent with a post-traumatic reaction of the tendon.

\section{Treatment}

Conservative measures are unlikely to succeed. Passive manipulation of the flexed thumb is painful 3 . and the condition returns at a later date. In one boy of five, such manipulation resulted in locking of the thumb in extension and operation was neces-o sary to relieve it. The condition when left untreated results in permanent flexion contracture, and a woman of $\mathbf{4 5}$ was seen with such a deformity giving a clear history of the condition existing since early $\sigma$ infancy.

Operative treatment is, therefore, advised. N Through a small transverse incision, the nodule is $O$ exposed and the digital nerves on each side of the

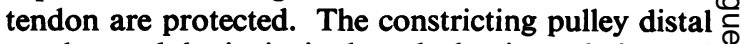
to the nodule is incised and the interphalangeal $\stackrel{\oplus}{\oplus}$ joint is extended fully. It has been found unneces- 0 sary to interfere with the nodule in the tendon. Only $\vec{O}$ the skin is sutured. 